\title{
IMPACT OF NOCTURIA ON SYMPTOM-SPECIFIC QUALITY OF LIFE AMONG COMMUNITY-DWELLING ADULTS AGED 40 YEARS AND OLDER
}

HONG-JENG YU, FONG-YING CHEN, PO-CHIEN HUANG, TONY HSIU-HSI CHEN, WEI-CHU CHIE, AND CHENG-YING LIU

\begin{abstract}
Objectives. To evaluate factors predicting the effect of nocturia in a community-based cohort of adults using a nocturia-specific quality-of-life (N-OOL) questionnaire.

Methods. From October 2004 to February 2005, adults aged 40 years old or older living in Matsu, Taiwan and reporting nocturia of one episode or more per night were interviewed with a 12-item N-OOL questionnaire consisting of Sleep/Energy and Bother/Concern subscales. Univariate analyses were used to analyze the effects of demographic characteristics, frequency and duration of nocturia, and sleeping characteristics on the $\mathrm{N}-\mathrm{OOL}$ score. Multiple linear regression analysis was used to identify factors predicting the $\mathrm{N}-\mathrm{QOL}$ score.

Results. A total of 663 adults completed this study (mean age 59.4 years). The average N-OOL scores (a lower score indicates worse $\mathrm{QOL}$ ) were $91.4 \pm 11.2,83.7 \pm 13.2,77.6 \pm 16.8$, and $67.6 \pm 21.2$ for nocturia episodes of $1,2,3$, and 4 or more per night, respectively $(P<0.001)$. Men reported significantly lower $\mathrm{N}-\mathrm{QOL}(85.6 \pm 15.1$ versus $88.9 \pm 13.1, P=0.003)$ and Bother/Concern subscale scores $(42.0 \pm 8.4$ versus $44.0 \pm 7.4, P<0.001)$, but not Energy/Sleep subscale scores $(43.6 \pm 7.8$ versus $44.4 \pm 7.3, P=$ 0.158 ) than women. On multiple linear regression analysis, increasing nocturia episodes (regression coefficient -6.2 , 95\% confidence interval -7.4 to -5.0 ), male sex (regression coefficient -3.5 , 95\% confidence interval -5.4 to -1.5 ), and degree of sleeping disturbance after nocturia (regression coefficient $-4.5,95 \%$ confidence interval -5.6 to -3.4 ) independently predicted a significantly lower N-OOL score.

Conclusions. The results of our study have confirmed that nocturia has a more significant QOL impact when the patient has two or more episodes per night. Moreover, men experienced a greater impact from nocturia than women, particularly in the Bother/Concern domain. UROLOGY 67: 713-718, 2006. () 2006 Elsevier Inc.
\end{abstract}

$\mathrm{N}$ octuria, defined by the International Continence Society Sub-Committee as a complaint that an individual has to wake from sleep to void, ${ }^{1}$ is one of the most bothersome lower urinary tract symptoms. ${ }^{2}$ It is a common reason for interrupted sleep in the general adult population ${ }^{3}$ and may subsequently result in adverse consequences such as a nighttime fall ${ }^{4}$ and a perception of poor health. ${ }^{5}$

From the Department of Urology, National Taiwan University Hospital and College of Medicine, Taipei, Taiwan; Hung Kuang University School of Nursing, Taichuang, Taiwan; Institute of Preventive Medicine, National Taiwan University College of Public Health, Taipei, Taiwan; and Health Bureau of Lienkiang County, Matsu, Taiwan

Reprint requests: Hong-Jeng Yu, M.D., Department of Urology, National Taiwan University Hospital, 7 Chungshan South Road, Taipei 100, Taiwan. E-mail: yhj5251@ha.mc.ntu.edu.tw

Submitted: August 19, 2005, accepted (with revisions): October 31, 2005
Although the great body of epidemiologic surveys has demonstrated nocturia to be a very prevalent urinary symptom, very little research has focused exclusively on the impact of nocturia on quality of life (QOL), which is a key predictor for healthcare seeking. In most questionnaire surveys, nocturia is included as one of several items the scores of which are added together to form a total, and, therefore, the impact of nocturia and its relationship to other factors is often difficult to discern. ${ }^{6}$ Currently, very few nocturia-specific constructed instruments have been specifically designed to investigate the impact of nocturia on various aspects of daily life.

The present study aimed to investigate the impact of nocturia on various aspects of daily life among community-dwelling adults using a nocturia-specific quality-of-life (N-QOL) questionnaire, an instrument developed and validated by Abra- 
ham and coworkers. ${ }^{7}$ Apart from the nocturia episodes, we attempted to analyze whether factors such as age, sex, and socioeconomic status had a significant association with the N-QOL scores.

\section{MATERIAL AND METHODS}

From October 2002 to February 2003, all inhabitants aged 30 years or older in Matsu, a small city located in the northeast of Taiwan, were invited to participate in a comprehensive healthcare-use screening (Matsu Community-Based Integrated Screening) supported by the Bureau of Health Promotion, Department of Health of Taiwan. A joint urologic survey designed to investigate the epidemiologic aspects of nocturia and overactive bladder syndrome were included as part of this study program. A total of 1706 adults completed this survey. The prevalence of nocturia and associated risk factors have been previously published. ${ }^{8}$ From October 2004 to February 2005 , those participants who were aged 40 years or older and reported one or more nocturia episodes per night in the survey ( $\mathrm{n}=682$ ) were interviewed again with the N-QOL questionnaire by public nurses. Of these, 74 were excluded either because of nonresponse $(n=53)$ or the reporting of no more nocturia $(\mathrm{n}=21)$. Additionally, we recruited 55 adults who had failed to participate in the previous survey and reported one or more nocturia episodes per night. Consequently, a total of 663 adults completed this study.

The details of the N-QOL questionnaire have been previously described. ${ }^{7}$ In brief, it consists of 12 items assessing the impact of nocturia on various aspects of daily life in the previous 4 weeks. Each item was scaled on a Likert-like response scale from 0 (least impact) to 4 (most impact). The first six items (Sleep/Energy subscale) cover the effect of nocturia on concentration, energy, napping, productivity, daily activities, and sleeping; and the subsequent six items (Bother/Concern subscale) cover the individual's bother and concern arising from nocturia, including concerns about drinking water, disturbing other family members, preoccupation with having to get up to urinate, worry that this condition would get worse, worry that no efficient treatment is available for this condition, and the global bother perceived from nocturia. For statistical analysis, the scores of these 12 items were summed and transformed into an N-QOL score from 0 to 100, with 100 indicating the best QOL. Also, the scores from the Sleep/Energy and Bother/Concern subscales were separately calculated ( 0 to 50 for each subscale). A pilot study that had included 50 adults ( 25 men and 25 women) was performed before the start of the interviews to ensure the validity of the questionnaire. A test and retest were conducted with a 2 -week interval between tests. The correlation coefficient was 0.87 for women, with a Cronbach coefficient alpha of 0.81 for the Sleep/Energy subscale and 0.84 for the Bother/Concern subscale. For men, the corresponding values were 0.88 , 0.80 , and 0.86

Potential factors that might affect the N-QOL, including demographic characteristics, nocturia episodes, duration of nocturia, total hours of sleep per night, and degree of sleeping disturbance after nocturia, were recorded. The degree of sleeping disturbance after nocturia was measured by asking the respondents how often they had difficulty in regaining sleep after waking up to void. The response was graded as 0 (never or less than one time in five), 1 (less than or about one half the time), and 2 (more than one half the time or almost always).

Continuous variables are presented as the mean \pm standard deviation and were compared using the $t$ test or analysis of variance. Categorical variables are presented as percentages and were compared using the chi-square test. Correlation analysis was performed using Spearman's rank correlation test. Univariate analysis was performed to evaluate the effect of potential variables on the N-QOL score. A multiple linear regression analysis model was constructed to test the independent effects of those variables that had a $P$ value of less than 0.1 in the univariate analysis. For all analyses, a $P$ value of less than 0.05 was considered statistically significant.

\section{RESULTS}

A total of 663 adults with a mean age of 59.4 years (range 40 to 79) were successfully interviewed. Of these adults, $56.9 \%, 28.4 \%, 10.6 \%$, and $4.2 \%$, reported one, two, three, and four or more nocturia episodes per night, respectively. After adjustment for age, the nocturia episodes correlated modestly with the N-QOL score $(r=-0.42, P<0.001)$ and weakly with the total hours of sleep $(r=-0.12$, $P=0.003$ ). The correlation was less significant with the degree of sleeping disturbance after nocturia ( $r=-0.07, P=0.053)$. In contrast, the NQOL score showed a modest correlation with the degree of sleeping disturbance after nocturia $(r=$ $-0.31, P<0.001)$ and a weaker correlation with the total hours of sleep $(r=0.13, P=0.003)$.

Table I gives the mean \pm standard deviation values for the Sleep/Energy, Bother/Concern, and N-QOL scores with respect to nocturia episodes, duration of nocturia, sex, age, educational level, employment, hours of sleep per night, and degree of sleeping disturbance after nocturia. The average N-QOL score was $91.4 \pm 11.2,83.7 \pm$ $13.2,77.6 \pm 16.8$, and $67.6 \pm 21.2$ for one, two, three, and four or more nocturia episodes per night, respectively $(P<0.001)$. On univariate analysis, significant differences in the N-QOL score were observed with regard to age $(P=0.006)$, sex $(P=0.003)$, total hours of sleep $(P=0.014)$, and degree of sleeping disturbance after nocturia $(P<$ $0.001)$, but not with regard to the duration of nocturia $(P=0.835)$, educational level $(P=0.059)$, or employment $(P=0.068)$.

Although no significant difference was found between men $(\mathrm{n}=335)$ and women $(\mathrm{n}=328)$ with respect to age $(59.6 \pm 11.8$ versus $59.3 \pm$ 12.3 years, $P=0.767$ ) or mean nocturia episodes ( $1.6 \pm 0.9$ versus $1.6 \pm 0.8, P=0.405)$, men had a significantly lower N-QOL score (85.6 \pm 15.1 versus $88.9 \pm 13.1, P=0.003)$ and Bother/Concern subscale score $(42.0 \pm 8.4$ versus $44.0 \pm 7.4$, $P<0.001)$. No significant difference was found in the Sleep/Energy subscale score (43.6 \pm 7.8 versus $44.4 \pm 7.3, P=0.158)$. When individual items were compared (Table II), men had a significantly lower score in the items "participate less in enjoyed activities" ( $P=0.046)$, "preoccupied about having to get up to urinate" $(P=0.006)$, "worried that the condition will get worse" $(P=$ 0.003), and "worried about no effective treatment" 
TABLE I. Effect of demographic, nocturia, and sleeping characteristics on $\mathrm{N}-\mathrm{OOL}$ score

\begin{tabular}{|c|c|c|c|c|}
\hline & Subjects (\%) & Sleep/Energy & Bother/Concern & N-QOL \\
\hline \multicolumn{5}{|l|}{ Nocturia episodes (n) } \\
\hline 1 & 377 (56.9) & $45.9 \pm 5.9$ & $45.4 \pm 6.2$ & $91.4 \pm 11.2$ \\
\hline 2 & $188(28.4)$ & $41.7 \pm 7.2$ & $41.9 \pm 7.3$ & $83.7 \pm 13.2$ \\
\hline 3 & $70(10.6)$ & $38.5 \pm 9.3$ & $39.0 \pm 9.1$ & $77.6 \pm 16.8$ \\
\hline$\geq 4$ & $28(4.2)$ & $35.7 \pm 12.4$ & $31.6 \pm 10.0$ & $67.6 \pm 21.2$ \\
\hline$P$ value (ANOVA) & & $<0.001$ & $<0.001$ & $<0.001$ \\
\hline \multicolumn{5}{|l|}{ Duration of nocturia (yr) } \\
\hline$<2$ & $213(32.1)$ & $44.1 \pm 7.5$ & $43.0 \pm 9.8$ & $87.2 \pm 14.1$ \\
\hline $2-4.9$ & $135(20.4)$ & $43.3 \pm 8.5$ & $43.3 \pm 7.6$ & $86.7 \pm 14.9$ \\
\hline $5-7.9$ & $141(21.3)$ & $44.0 \pm 7.7$ & $42.8 \pm 7.8$ & $86.8 \pm 14.6$ \\
\hline$\geq 8$ & $174(26.2)$ & $44.4 \pm 6.6$ & $43.4 \pm 9.8$ & $88.0 \pm 13.6$ \\
\hline$P$ value (ANOVA) & & 0.655 & 0.892 & 0.835 \\
\hline \multicolumn{5}{|l|}{ Sex } \\
\hline Men & 335 (50.5) & $43.6 \pm 7.8$ & $42.0 \pm 8.4$ & $85.6 \pm 15.1$ \\
\hline Women & $328(49.5)$ & $44.4 \pm 7.3$ & $44.0 \pm 7.4$ & $88.9 \pm 13.1$ \\
\hline$P$ value ( $t$ test) & & 0.158 & $<0.001$ & 0.003 \\
\hline \multicolumn{5}{|l|}{ Age $(y r)$} \\
\hline $40-49$ & $187(28.2)$ & $45.1 \pm 6.9$ & $44.3 \pm 7.2$ & $89.4 \pm 12.9$ \\
\hline $50-59$ & $164(24.7)$ & $44.9 \pm 6.3$ & $43.7 \pm 7.4$ & $88.6 \pm 12.5$ \\
\hline $60-69$ & $143(21.6)$ & $42.9 \pm 8.0$ & $42.7 \pm 7.4$ & $85.6 \pm 14.6$ \\
\hline $70+$ & 169 (25.5) & $42.8 \pm 8.6$ & $41.8 \pm 8.9$ & $84.9 \pm 16.4$ \\
\hline$P$ value (ANOVA) & & 0.004 & 0.014 & 0.006 \\
\hline \multicolumn{5}{|l|}{ Employed } \\
\hline No & $303(45.7)$ & $43.1 \pm 8.1$ & $42.8 \pm 8.1$ & $86.1 \pm 14.8$ \\
\hline Yes & $360(54.3)$ & $44.6 \pm 7.0$ & $43.4 \pm 7.5$ & $88.1 \pm 13.6$ \\
\hline$P$ value $(t$ test $)$ & & 0.010 & 0.286 & 0.068 \\
\hline \multicolumn{5}{|l|}{ Education } \\
\hline Primary school or below & $268(40.4)$ & $42.7 \pm 8.6$ & $42.7 \pm 8.5$ & $85.5 \pm 15.9$ \\
\hline Middle to high school & $294(44.3)$ & $44.6 \pm 6.8$ & $44.0 \pm 7.0$ & $88.7 \pm 12.8$ \\
\hline College or above & $101(15.3)$ & $44.2 \pm 7.2$ & $42.6 \pm 8.1$ & $87.1 \pm 14.1$ \\
\hline$P$ value (ANOVA) & & 0.017 & 0.106 & 0.059 \\
\hline \multicolumn{5}{|l|}{ Sleep $(h r)$} \\
\hline$<6$ & $146(22.0)$ & $42.1 \pm 9.1$ & $42.3 \pm 9.6$ & $84.4 \pm 17.2$ \\
\hline$\geq 6$ to $<8$ & $413(62.3)$ & $44.2 \pm 7.1$ & $43.3 \pm 7.3$ & $87.7 \pm 13.5$ \\
\hline$\geq 8$ & $104(15.7)$ & $45.6 \pm 6.2$ & $43.8 \pm 6.9$ & $89.4 \pm 11.8$ \\
\hline$P$ value (ANOVA) & & 0.001 & 0.253 & 0.014 \\
\hline \multicolumn{5}{|c|}{ Degree of sleep disturbance after nocturia* } \\
\hline 0 & $291(43.9)$ & $46.4 \pm 6.0$ & $45.4 \pm 6.0$ & $91.8 \pm 11.1$ \\
\hline 1 & $163(24.6)$ & $43.1 \pm 7.6$ & $42.0 \pm 8.4$ & $85.3 \pm 14.8$ \\
\hline 2 & $209(31.5)$ & $41.0 \pm 8.3$ & $40.6 \pm 8.6$ & $81.7 \pm 15.7$ \\
\hline$P$ value (ANOVA) & & $<0.001$ & $<0.001$ & $<0.001$ \\
\hline
\end{tabular}

KEY: N-QOL = nocturia-specific quality of life (questionnaire); ANOVA = analysis of variance.

* Degree of sleep disturbance after nocturia: 0, rare or less than one time in five; 1, less than one half to about one half the time; 2 , more than one half the time or almost always.

$(P=0.000)$. Only marginal significance was observed for the item "bothersomeness arising from nocturia" $(P=0.062)$.

Multiple linear regression analysis was then used to test the independent effects of potential factors on the N-QOL score. The results demonstrated that increasing numbers of nocturia episodes (regression coefficient $-6.2,95 \%$ confidence interval [CI] -7.4 to -5.0 ), male gender (regression coefficient $-3.5,95 \% \mathrm{CI}-5.4$ to -1.5 ), and degree of sleeping disturbance after nocturia (regression coefficient $-4.5,95 \% \mathrm{CI}-5.6$ to -3.4 ) were independent factors predicting a significantly lower NQOL score (Table III).

\section{COMMENT}

In the present study, the N-QOL measurement was chosen because it has been shown to have good psychometric validity for use in men with nocturia. ${ }^{4}$ Our pilot study using this questionnaire in both sexes also demonstrated good internal consistency and good test-retest reliability. Moreover, the brevity and easy understanding of this instrument enabled us to use it in our study cohort in which $32 \%$ of the participants were aged 65 years or older. According to our previous experience, ${ }^{9}$ many older respondents may get tired and impatient with a lengthy questionnaire, which could 
TABLE II. Comparison of mean scores for individual items on $\mathrm{N}-\mathrm{OOL}$ between men and women

\begin{tabular}{|c|c|c|c|}
\hline \multirow[b]{2}{*}{ Item } & \multicolumn{2}{|c|}{ Mean Score* } & \multirow{2}{*}{$\begin{array}{l}\text { Adjusted } \\
P \text { Value }\end{array}$} \\
\hline & Men & Women & \\
\hline \multicolumn{4}{|l|}{ Sleep/Energy } \\
\hline Difficult to concentrate the next day & $3.30 \pm 0.90$ & $3.32 \pm 0.94$ & 0.554 \\
\hline Low in energy the next day & $3.54 \pm 0.69$ & $3.59 \pm 0.72$ & 0.286 \\
\hline Less productive the next day & $3.50 \pm 0.75$ & $3.57 \pm 0.69$ & 0.999 \\
\hline Participate less in enjoyed activities & $3.50 \pm 0.80$ & $3.62 \pm 0.70$ & 0.046 \\
\hline Require nap during the day & $3.56 \pm 0.74$ & $3.64 \pm 0.70$ & 0.227 \\
\hline Difficult to get enough sleep at night & $3.22 \pm 0.94$ & $3.32 \pm 0.87$ & 0.243 \\
\hline \multicolumn{4}{|l|}{ Bother/Concern } \\
\hline Careful about drinking water & $3.21 \pm 1.00$ & $3.28 \pm 0.97$ & 0.148 \\
\hline Concern about disturbing others & $3.62 \pm 1.01$ & $3.71 \pm 0.78$ & 0.122 \\
\hline $\begin{array}{l}\text { Preoccupied about having to get up to } \\
\text { urinate }\end{array}$ & $3.40 \pm 0.85$ & $3.58 \pm 0.77$ & 0.006 \\
\hline Worried that the condition will get worse & $3.32 \pm 0.90$ & $3.53 \pm 0.81$ & 0.003 \\
\hline Worried about no effective treatment & $3.30 \pm 0.95$ & $3.59 \pm 0.77$ & 0.000 \\
\hline Bothersomeness arising from nocturia & $3.28 \pm 0.83$ & $3.41 \pm 0.80$ & 0.062 \\
\hline \multicolumn{4}{|c|}{$\begin{array}{l}\text { KEY: } N-Q O L=\text { nocturia-specific quality of life (questionnaire). } \\
\text { * Scores range from } 0 \text { to } 4: 0 \text {, "extremely bothersome"; } 4 \text {, "not bothersome at all." } \\
\text { " Adjustment for age and nocturia episodes. }\end{array}$} \\
\hline
\end{tabular}

TABLE III. Association of factors with N-OOL score on multiple linear regression analysis

\begin{tabular}{lcccc}
\hline Variable & $\begin{array}{c}\text { Regression } \\
\text { Coefficient }\end{array}$ & $\boldsymbol{P}$ Value & $\mathbf{9 5 \%} \mathbf{C l}$ & Tolerance \\
\hline Age (per 10 yr) & 0.2 & 0.694 & & 0.68 \\
Male gender & -3.5 & 0.000 & -5.4 to -1.5 & 0.90 \\
Nocturia episodes & -6.2 & 0.000 & -7.4 to -5.0 & 0.93 \\
Degree of sleep disturbance & -4.5 & 0.000 & -5.6 to -3.4 & 0.93 \\
$\quad$ after nocturia & 0.6 & 0.610 & & 0.71 \\
Job (yes/no) & 0.2 & 0.741 & & 0.77 \\
Education level & 1.1 & 0.213 & & 0.95 \\
Total hours of sleep & & & \\
KEY: N-QOL = nocturia-specific quality of life (questionnaire); CI = confidence interval. &
\end{tabular}

result in a substantial bias on the outcome measurements. Our results have shown that nocturia in community-dwelling adults appears to have a significant impact on daily activities, including sleep, vitality, and emotional health. The QOL impact caused by nocturia was more significant when patients experienced two or more nocturia episodes per night. In addition, men experienced a greater impact from nocturia than women, particularly in the Bother/Concern domain.

The number of nocturia episodes was undoubtedly the most important factor contributing to the impact on QOL. In the present study, multiple linear regression analysis showed that as the number of nocturia voids increased by one, the N-QOL score decreased by 6.2 (95\% CI -7.4 to -5.0$)$. Several surveys from community ${ }^{10-12}$ and clinical settings ${ }^{13,14}$ have suggested that individuals with two or more nocturia episodes per night are more likely to perceive bother. In agreement with these reports, our study showed that the average N-QOL score was greater than 90 for those who reported only one nocturia episode per night, indicating less QOL effect.

The detrimental effect of nocturia on sleep is well recognized. Middlekoop et al. ${ }^{3}$ reported that $67.5 \%$ of their community sample claimed nocturia to be the most frequent cause of sleeping disturbance. In a survey of 5204 community-dwelling people in the United States, Coyne et al. ${ }^{12}$ demonstrated a significant correlation between nocturia episodes and the number of hours of sleep. In the present study, the relationship between nocturia episodes and total hours of sleep was less obvious. The correlation between the total hours of sleep and nocturia episodes was weak $(r=-0.12, P=0.003)$, and the factor of the total hours of sleep was not significantly predictive of the N-QOL score in the multiple linear regression analysis. We found great individual differences with respect to the degree 
of sleep disturbance after nocturia: $31.5 \%$ versus $43.9 \%$ of the individuals reported having great or having little sleep disturbance after nocturia (Table II), and this factor appeared to be important for predicting a lower N-QOL score (regression coefficient $-4.5,95 \%$ CI -5.6 to -3.4 ).

Studies comparing men and women with respect to the perception of how bothersome nocturia are limited. By using a global bother question, Schatzl et al. ${ }^{10}$ reported that $66.9 \%$ of women and $62.2 \%$ of men with nocturia reported a QOL impairment. In contrast, a study in a Danish population showed that women were more bothered by nocturia than were men. ${ }^{15}$ The use of the N-QOL score in the present study provided a quantitative measure for comparing sexual difference in QOL impacts from nocturia. Men reported a significantly lower N-QOL score than women and subscale analyses revealed that the difference mainly stemmed from a lower Bother/Concern score rather than a lower Sleep/Energy score. This result implies that although nocturia has similar effects on sleep and vitality in both sexes, men are more concerned and bothered by nocturia. The reasons for this sexual difference are unclear. It might be that men are more likely to link their urinary symptoms to benign prostatic hyperplasia, and hence, increasing nocturia episodes may be perceived as a deterioration of the prostate condition. In contrast, more tolerance of nocturia in women could result from social acceptability, because our previous study has shown that older women are more likely to believe that their urinary symptoms are an inevitable consequence of aging. ${ }^{9}$

The effect of age on the QOL impact from nocturia was also explored in the present study. Although the N-QOL score decreased significantly across age groups in univariate analysis, age was not an independent factor on multivariate analysis. Because nocturia episodes increased significantly with age, this result might suggest that the decrease in N-QOL score among the elderly stems from increasing nocturia episodes rather than from aging. Similarly, other studies have also demonstrated that the bothersomeness associated with lower urinary tract symptoms, including nocturia, is relatively unrelated to age. ${ }^{12,13}$ Also, although the impact of nocturia on professionally active individuals has been documented, ${ }^{16}$ professional working appeared not to be a significant factor predicting greater QOL impact in the present study.

One interesting finding in the present study was that the average N-QOL score for each nocturia episode appeared to be greater than that obtained from another study using an identical questionnaire. ${ }^{7}$ Because QOL is an abstract and highly subjective concept influenced by personal and cultural values, age, and life expectancy, it is not known whether culture plays a role in the perception of nocturia.

The possible limitations of this study need to be discussed. Young adults (younger than 40 years) were not studied because of a greater rate of not responding owing to working or not living in town on weekdays. Moreover, the city of Matsu is a small community and, therefore, the possibility of a cohort effect must be ruled out in a comparative study between different communities. Finally, because the N-QOL was initially developed using input from male patients, the data concerning sexual differences in the N-QOL score should be interpreted with caution, although our study confirmed its validity in women.

\section{CONCLUSIONS}

Nocturia has a negative impact on various aspects of daily life of community-dwelling adults, including sleeping, vitality, and emotional health. Our results indicate that, apart from the increasing number of nocturia episodes, men and women with sleeping disturbance after nocturia experience a greater effect from nocturia. Because nocturia is one of the most common urinary symptoms in the elderly, who represent the fastest growing population group in many countries, it is likely to have a significant impact on the health services of such countries.

\section{REFERENCES}

1. Van Kerrebroeck P, Abrams P, Chaikin D, et al: The standardization of terminology in nocturia: report from the Standardization Subcommittee of the International Continence Society. Neurourol Urodyn 21: 179-183, 2002.

2. Weiss JP, and Blaivas JG: Nocturia. J Urol 163: 5-12, 2000.

3. Middlekoop HA, Smlide-van den Doel DA, Neven AK, et al: Subjective sleep characteristics of 1485 males and females aged 59-93: effects of sex and age, and factors related to self-evaluated quality of sleep. J Gerontol A Biol Sci Med Sci 51: M108-M115, 1996.

4. Stewart RB, Moore MT, May FE, et al: Nocturia: a risk factor for falls in the elderly. J Am Geriatr Soc 40: 1217-1220, 1992.

5. Asplund R, and Aberg HE: Nocturia and health in women aged 40-64 years. Maturitas 35: 143-148, 2000.

6. Donovan JL: Measuring the impact of nocturia on quality of life. BJU Int 84(suppl 1): 21-25, 1999.

7. Abraham L, Hareendran A, Mills IW, et al: Development and validation of a quality-of-life measure for men with nocturia. Urology 63: 481-486, 2004.

8. Yu HJ, Chen TH, Chie WC, et al: Prevalence and associated factors of nocturia among adult residents of the Matsu area of Taiwan. J Formos Med Assoc 104: 444-447, 2005.

9. Yu HJ, Wong WY, Chen J, et al: Quality of life impact and treatment seeking of Chinese women with urinary incontinence. Qual Life Res 12: 327-333, 2003.

10. Schatzl G, Temml C, Schmidbauer J, et al: Cross-sectional study of nocturia in both sexes: analysis of a voluntary health screening project. Urology 56: 71-75, 2000.

11. Swithinbank LV, Donovan JL, du Heaume JC, et al: Urinary symptoms and incontinence in women: relationships 
between occurrence, age, and perceived impact. Br J Gen Pract 49: 897-900, 1999.

12. Coyne KS, Zhou Z, Bhattacharyya SK, et al: The prevalence of nocturia and its effect on health-related quality of life and sleep in a community sample in the USA. BJU Int 92: 948-954, 2003.

13. Peters TJ, Donovan JL, Kay HE, et al: The International Continence Society "Benign Prostatic Hyperplasia" study: the bothersomeness of urinary symptoms. J Urol 157: 885-889, 1997.
14. Fiske J, Scarpero HM, Xue X, et al: Degree of bother caused by nocturia in women. Neurourol Urodyn 23: 130133, 2004.

15. Kay L, Stigsby B, Brasso K, et al: Lower urinary tract symptoms-a population survey using the Danish Prostatic Symptom Score (DAN-PSS) questionnaire. Scand J Urol Nephrol 33: 94-99, 1999.

16. Kobelt G, Borgstrom F, and Mattiasson A: Productivity, vitality and utility in a group of healthy professionally active individuals with nocturia. BJU Int 91: 190-195, 2003. 